\title{
Anisotropic stars as ultracompact objects in general relativity
}

\author{
Guilherme Raposo, ${ }^{1}$ Paolo Pani, ${ }^{1}$ Miguel Bezares, ${ }^{2}$ Carlos Palenzuela, ${ }^{2}$ and Vitor Cardoso ${ }^{3,4}$ \\ ${ }^{1}$ Dipartimento di Fisica, "Sapienza" Università di Roma \& Sezione INFN Romal, \\ P.A. Moro 5, 00185 Roma, Italy \\ ${ }^{2}$ Departament de Física IAC3, Universitat de les Illes Balears and Institut d'Estudis Espacials de \\ Catalunya, Palma de Mallorca, Baleares E-07122, Spain \\ ${ }^{3}$ CENTRA, Departamento de Física, Instituto Superior Técnico-IST, Universidade de Lisboa-UL, \\ Avenida Rovisco Pais 1, 1049 Lisboa, Portugal \\ ${ }^{4}$ Theoretical Physics Department, CERN 1 Esplanade des Particules, Geneva 23 CH-1211, Switzerland
}

(Received 22 November 2018; published 28 May 2019)

\begin{abstract}
Anisotropic stresses are ubiquitous in nature, but their modeling in general relativity is poorly understood and frame dependent. We introduce the first study on the dynamical properties of anisotropic self-gravitating fluids in a covariant framework. Our description is particularly useful in the context of tests of the black hole paradigm, wherein ultracompact objects are used as black hole mimickers but otherwise lack a proper theoretical framework. We show the following: (i) anisotropic stars can be as compact and as massive as black holes, even for very small anisotropy parameters; (ii) the nonlinear dynamics of the $1+1$ system is in good agreement with linearized calculations, and shows that configurations below the maximum mass are nonlinearly stable; (iii) strongly anisotropic stars have vanishing tidal Love numbers in the black-hole limit; and (iv) their formation will usually be accompanied by gravitational-wave echoes at late times.
\end{abstract}

DOI: 10.1103/PhysRevD.99.104072

\section{INTRODUCTION}

A foundational result in general relativity (GR) states that the maximum compactness of a self-gravitating, isotropic, spherically symmetric object of mass $M$ and radius $R$ is $M / R=4 / 9$, if the object is composed of a perfect fluid [1] (we use $G=c=1$ units). As a corollary, under the assumptions above, the existence of exotic compact objects (ECOs) of compactness arbitrarily close to that of a Schwarzschild black hole $(\mathrm{BH}$, with $M / R=1 / 2$ ) is ruled out. Thus, tests of the $\mathrm{BH}$ paradigm - are the dark and massive objects that we see really BHs? - are challenging to devise, impacting our ability to quantify statements about evidence for BHs [2-6], or to discover new species of compact objects.

It has been realized that Buchdahl's bound above relies strongly on the hypothesis of isotropy. Anisotropies in matter fields arise naturally at high densities [7-9] and may play an important role in the interior of compact objects. The simplest known example is that of a scalar field minimally coupled to gravity, which indeed gives rise to

Published by the American Physical Society under the terms of the Creative Commons Attribution 4.0 International license. Further distribution of this work must maintain attribution to the author(s) and the published article's title, journal citation, and DOI. anisotropic pressure in boson stars [10]. Other examples include electromagnetic fields, fermionic fields, pioncondensed phase configurations in neutron stars [11], superfluidity [12], solid cores [7], etc. In the real world, anisotropic pressures are the rule rather than the exception.

Surprisingly, anisotropic stars in GR are poorly studied. While various solutions have been obtained, both in closed form [13-20] and numerically [21-27], none arise from a consistent covariant model (see Ref. [28] for some progress). The lack of a proper framework prevents the exploration of outstanding questions associated with these objects, such as their stability, dynamical formation, and phenomenology. This is in stark contrast with the excellent knowledge on the dynamics of BHs and neutron stars, and is also the most important limitation in the study of ECOs $[2,3,6]$ (the only exception being boson stars which, however, are even less compact than Buchdahl's bound [10] and do not belong to the ClePhOs category introduced in Refs. [2,3,6]).

Here we introduce a covariant and self-consistent model for anisotropic fluids in GR, admitting stable and wellbehaved ultracompact solutions which we term $\mathcal{C}$-stars.

For the sake of simplicity, we will mostly restrict our analysis to the spherically symmetric case; a covariant extension to the general case (without spherical symmetry) is provided in Appendix A. 


\section{COVARIANT APPROACH TO ANISOTROPIES}

Consider an anisotropic fluid with radial pressure $P_{r}$, tangential pressure $P_{t}$, and total energy density $\rho$, described by the stress-energy tensor $[13,22]$

$$
T_{\mu \nu}=\rho u_{\mu} u_{\nu}+P_{r} k_{\mu} k_{\nu}+P_{t} \Pi_{\mu \nu},
$$

where $u_{\mu}$ is the fluid four-velocity and $k_{\mu}$ is a unit spacelike vector orthogonal to $u_{\mu}$, i.e., $k^{\mu} k_{\mu}=1=-u^{\mu} u_{\mu}$, $u^{\mu} k_{\mu}=0$. Here, $\Pi_{\mu \nu}=g_{\mu \nu}+u_{\mu} u_{\nu}-k_{\mu} k_{\nu}$ is a projection operator onto a two-surface orthogonal to $u^{\mu}$ and $k^{\mu}$, i.e., $u_{\mu} \Pi^{\mu \nu} V_{\nu}=k_{\mu} \Pi^{\mu \nu} V_{\nu}=0$ for any vector $V^{\mu}$.

At the center of symmetry of the fluid the anisotropy $P_{r}-P_{t}$ must vanish [13]. There is a certain degree of arbitrariness to satisfy this condition in a covariant fashion: the simplest possibility is

$$
P_{t}=P_{r}-\mathcal{C} f(\rho) k^{\mu} \nabla_{\mu} P_{r}
$$

where $f(\rho)$ is a generic function of the density and the free constant $\mathcal{C}$ is a parameter that measures the deviation from isotropy. For example, for the case $f(\rho)=\rho$ considered below, $\mathcal{C}$ has dimensions length cubed and Eq. (2) shows that the density scale at which $P_{t}-P_{r} \gg P_{r}$ is $\rho \gg \rho_{\text {anis }}$ with

$$
\rho_{\text {anis }} \sim \frac{R}{\mathcal{C}} \sim 6 \times 10^{15}\left(\frac{10^{4}}{\overline{\mathcal{C}}}\right)\left(\frac{R}{M}\right)\left(\frac{M}{M_{\odot}}\right) \frac{\mathrm{g}}{\mathrm{cm}^{3}},
$$

where $\overline{\mathcal{C}}=\mathcal{C} / M_{\odot}^{3}$ and we have identified a typical length scale with the radius $R$. By construction, $P_{t}=P_{r}$ at the center of static and spherically symmetric objects, since $\left.\partial_{r} P_{r}\right|_{r=0}=0$.

By defining $\sigma:=f(\rho) k^{\mu} \nabla_{\mu} P_{r}$, we can write Eq. (1) as the stress-energy tensor of an isotropic perfect fluid plus an anisotropic contribution,

$$
T_{\nu}^{\mu}=\left(\rho+P_{r}\right) u^{\mu} u_{\nu}+P_{r} g_{\nu}^{\mu}-\mathcal{C} \sigma \Pi_{\nu}^{\mu} .
$$

In the spherically symmetric case, $u^{\mu}=\left(u^{0}, u^{1}, 0,0\right)$, $k^{\mu}=\left(k^{0}, k^{1}, 0,0\right)$, and all dynamical variables are functions of $(t, r)$ only. The orthogonality conditions provide two constraints on $k^{\mu}$, which is therefore completely fixed in terms of $u^{\mu}$. It is straightforward to show that $\Pi_{\nu}^{\mu}=$ $\operatorname{diag}(0,0,1,1)$, which simplifies some of the computations presented below.

\section{III. $\mathcal{C}$-STARS: EQUILIBRIUM CONFIGURATIONS}

For static solutions, the metric can be written as $d s^{2}=$ $-e^{\nu(r)} d t^{2}+(1-2 m(r) / r)^{-1} d r^{2}+r^{2}\left(d \theta^{2}+\sin ^{2} \theta d \varphi^{2}\right)$. The metric variables satisfy the standard relation, $m^{\prime}(r)=$ $4 \pi r^{2} \rho$ and $\nu^{\prime}(r)=2\left(m+4 \pi r^{3} P_{r}\right) /(r(r-2 m))$, whereas the radial pressure satisfies a modified Tolman-OppenheimerVolkoff equation,

$$
P_{r}^{\prime}(r)=-\frac{\left(P_{r}+\rho\right)}{r(r-2 m)} \frac{\left(m+4 \pi r^{3} P_{r}\right)}{1+\frac{2}{r} \mathcal{C} f(\rho) \sqrt{1-\frac{2 m}{r}}},
$$

which reduces to the isotropic case when $\mathcal{C}=0$.

Two equations of state for $P_{r}$ and $f(\rho)$ are necessary to close the system. The simplest choice for the function $f$ would be $f(\rho)=1$, but in this model $P_{t}^{\prime}$ is discontinuous at the stellar radius since $P_{t}^{\prime}(R) \neq 0$; see Eq. (2). The simplest model that ensures the continuity of $P_{t}$ and its derivative at the radius is $f(\rho)=\rho$. We focus on this case here, although other models [e.g., $f(\rho)=\rho^{n}, n>0$ ] give similar results. With this choice, Eqs. (5) and (2) guarantee that $P_{r}=$ $P_{r}^{\prime}=P_{t}=P_{t}^{\prime}=0$ at $r=R$.

Remarkably, Eq. (5) can be solved in closed form for a toy model of an incompressible fluid $[\rho(r)=$ const $]$, although the solution is cumbersome. We focus instead on a standard polytropic equation of state $P_{r}=K \rho_{0}^{\gamma}$ with adiabatic index $\gamma=2$, where $\rho_{0}=\rho-P_{r} /(\gamma-1)$ is the rest-mass density. Our results are qualitatively the same for other standard equations of state.

The mass-radius diagram and fluid profiles of $\mathcal{C}$-stars are shown respectively in Figs. 1 and 2, for different values of

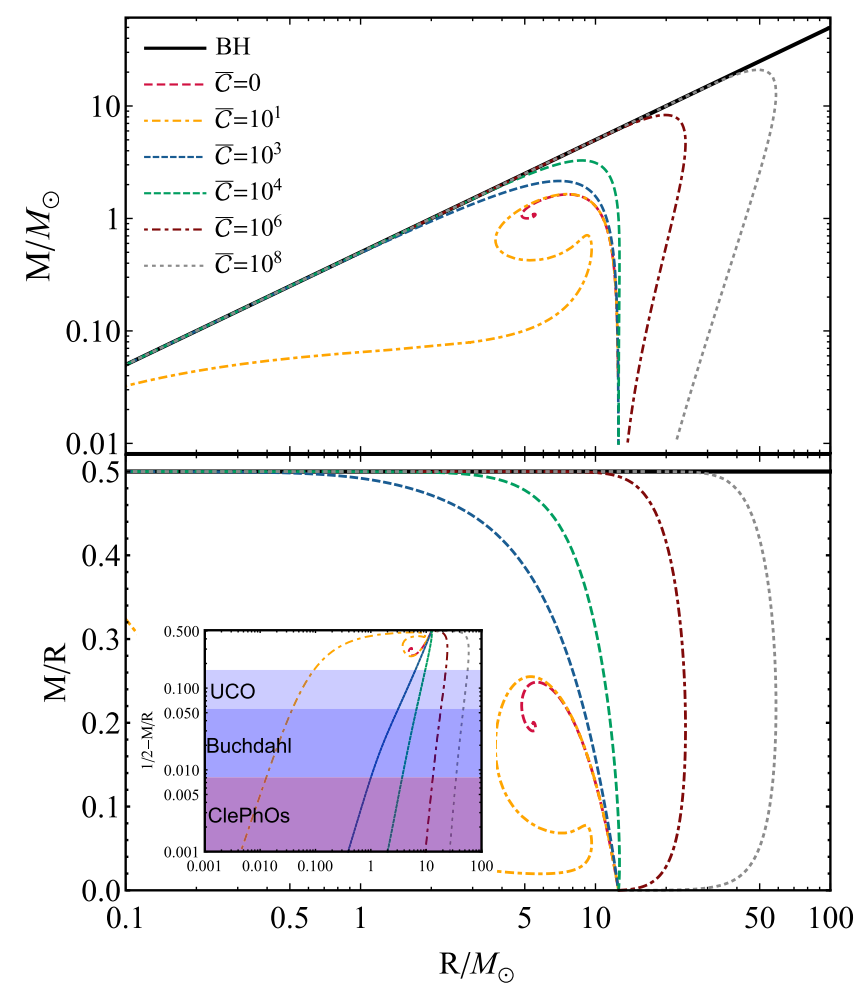

FIG. 1. Mass-radius and compactness diagrams for $\mathcal{C}$-stars with various values of the (dimensionless) anisotropy parameter $\overline{\mathcal{C}}$. Note that when $\overline{\mathcal{C}}$ is small the $M-R$ diagram shows peculiar turning points, which "open up" in the large- $\overline{\mathcal{C}}$ limit. The inset shows the deviation $1 / 2-M / R$ from the compactness of a Schwarzschild $\mathrm{BH}$ on a logarithmic scale. $\mathcal{C}$-stars exist across the various categories (UCOs, ClePhOs) introduced in Refs. [2,3]. 


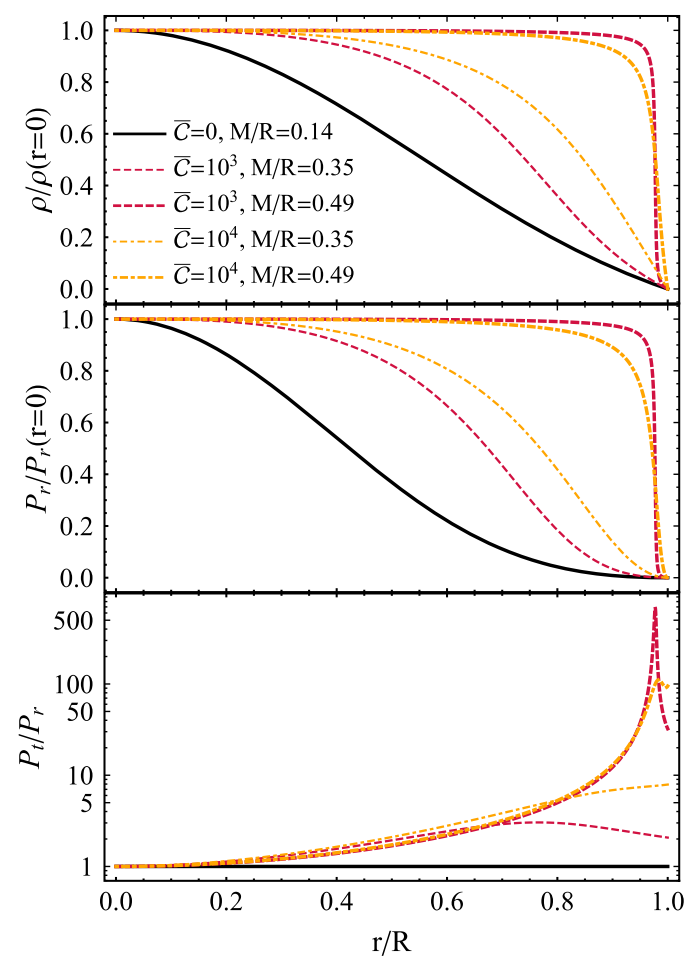

FIG. 2. Energy density (top panel), radial pressure (middle panel), and tangential pressure (bottom panel) profiles for different configurations of $\mathcal{C}$-stars with different anisotropy parameters and compactness. The density and radial pressure are normalized by the corresponding values at the center, while the tangential pressure is normalized with the radial pressure at the same radius. The black solid line represents an isotropic configuration with $M / R=0.14$, the dashed thin (thick) red lines correspond to anisotropic configurations with $\overline{\mathcal{C}}=10^{3}$ for $M / R=0.35$ $(M / R=0.49)$, and the dot-dashed thin (thick) yellow lines correspond to anisotropic configurations with $\overline{\mathcal{C}}=10^{4}$ for $M / R=0.35(M / R=0.49)$. The results indicate that the qualitative behavior of the fluid variables in this model is roughly independent of $\overline{\mathcal{C}}$ but depends on the compactness of the configuration. As $M / R \rightarrow 0.5$, the star tends to a constant-density configuration, while the tangential pressure profile tends to have a very sharp peak near the radius of the star.

$\mathcal{C}$, related to the density scale of strong anisotropies by Eq. (3). There are several features worth highlighting. (i) Overall, $\mathcal{C}$-stars can be much more compact and massive than isotropic stars and their maximum compactness always approaches that of a Schwarzschild $\mathrm{BH}$ in some region, when $\rho \gg \rho_{\text {anis }}$. (ii) More importantly, $\mathcal{C}$-stars exist across a wide range of masses, evading one of the outstanding issues with BH mimickers: in most theories giving rise to ECOs, these approach the $\mathrm{BH}$ compactness in a very limited range of masses, thus being unable to describe both stellar-mass and supermassive BH candidates across several orders of magnitude in mass. On the other hand, $\mathcal{C}$-stars can do so when $\mathcal{C} / M^{3} \gg 1$. (iii) As shown in the inset of Fig. 1, compact configurations exceed Buchdahl's limit and can even be classified as ClePhOs in the classification of
TABLE I. Properties of some representative $\mathcal{C}$-star solutions. The last column presents the values of $\bar{\sigma}_{\max }:=\max \left\{\left(P_{t}-P_{r}\right) /\right.$ $\left.P_{r}\right\}$, which gives a measurement of the maximum anisotropy in the interior of the star. Anisotropies are moderate for mildly compact configurations, whereas more compact configurations exhibit larger anisotropies, as also shown in the bottom panel of Fig. 2.

\begin{tabular}{lcccc}
\hline \hline$\overline{\mathcal{C}}$ & $\rho_{c}\left(\times 10^{15} \mathrm{gcm}^{3}\right)$ & $R / M_{\odot}$ & $M / M_{\odot}$ & $\bar{\sigma}_{\max }$ \\
\hline $10^{3}$ & 2.42 & 5.97 & 2.09 & 2.04 \\
$10^{3}$ & 3.70 & 4.46 & 1.80 & 4.66 \\
$10^{3}$ & 17.0 & 1.11 & 0.54 & 682 \\
$10^{4}$ & 0.90 & 9.28 & 3.25 & 6.90 \\
$10^{4}$ & 1.20 & 8.04 & 3.24 & 11.4 \\
$10^{4}$ & 3.50 & 3.84 & 1.88 & 110 \\
\hline \hline
\end{tabular}

Refs. [2,3]. (iv) In general, the qualitative behavior of the equilibrium solutions depends only mildly on $\mathcal{C}$, while it depends strongly on the compactness of the star. This is shown in Fig. 2. Configurations with moderately low compactness display fluid profiles qualitatively similar to the isotropic case. However, as the compactness increases and approaches the black-hole compactness, the radial pressure and density profiles tend to constant values in the stellar interior, while the anisotropy $P_{t}-P_{r}$ vanishes in the core and displays a very sharp peak close to the radius of the star. In the $M / R \rightarrow 0.5$ limit this peak becomes infinitesimally thin and approaches the radius of the star, in a way reminiscent of gravastars with a thin layer of strongly anisotropic pressure [29-32]. For comparison, in Table I we present data for different representative configurations.

(v) When $\mathcal{C} \geq 0$ the fluid has $P_{t}>0$ everywhere inside the star, and satisfies the weak and strong energy conditions [33] $\left(\rho+P_{r}+2 P_{t} \geq 0, \rho+P_{r} \geq 0\right.$, and $\left.\rho+P_{t} \geq 0\right)$, whereas very compact configurations violate the dominant energy condition $\left(\rho \geq P_{r}\right.$ and $\left.\rho \geq P_{t}\right)$ near the radius, where $P_{t}$ attains a maximum and $P_{t}>\rho$, for very compact configurations.

\section{RADIAL STABILITY}

For any $\mathcal{C}>0$ the compactness and the anisotropy grow in the high-density region, eventually reaching the $\mathrm{BH}$ compactness (see inset of Fig. 1). Thus, even a vanishingly small value of the anisotropy parameter $\mathcal{C}$ can give rise to strongly anisotropic quasi-Schwarzschild equilibrium solutions. When $\mathcal{C}$ is small, standard analysis of the turning points in the mass-radius diagram [34] suggests that these configurations are unstable. On the other hand, in the strong-anisotropy regime, the mass-radius relation of a $\mathcal{C}$-star approaches that of a $\mathrm{BH}$ already on the stable branch. To test these issues, we perform a linear stability analysis of $\mathcal{C}$-stars under radial perturbations. The spacetime metric is written as $g_{\mu \nu}=g_{\mu \nu}^{(0)}+h_{\mu \nu}$, where $g_{\mu \nu}^{(0)}$ is the metric of a background $\mathcal{C}$-star solution and $h_{\mu \nu}=\operatorname{diag}\left(H_{0}(r)\right.$, $\left.H_{2}(r), 0,0\right) e^{-i \omega t}$ is a small perturbation in Fourier space. Likewise, we expand the fluid density, pressure, and vector 


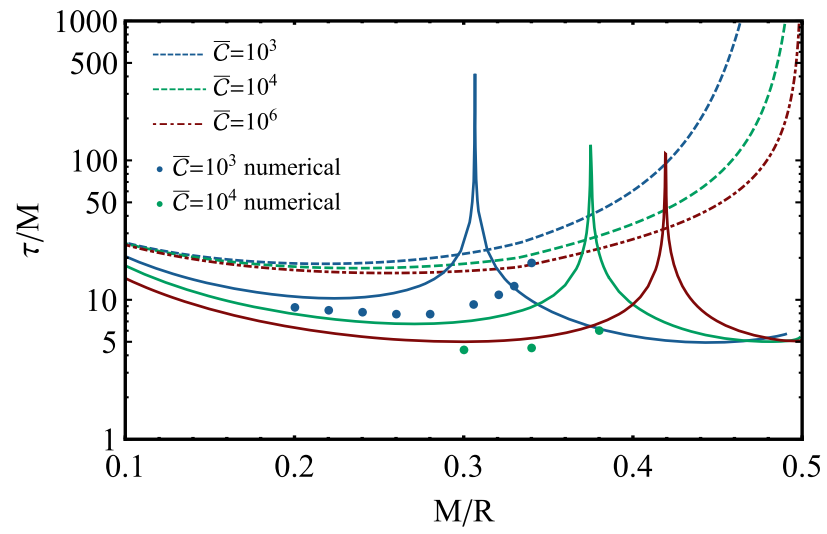

FIG. 3. The time scale $\tau=1 /|\omega|$ for $\mathcal{C}$-stars as a function of the compactness for various values of $\overline{\mathcal{C}}$ (continuous curves). Configurations on the left of the cusps (corresponding to the zero crossing of $\omega^{2}$ ) are linearly stable, whereas those on the right are linearly unstable. The threshold corresponds to the maximum mass of the object shown in Fig. 1. We also show the echo delay time (15) for these configurations (dashed curves). The markers refer to the time scale extracted from the nonlinear evolutions, which systematically predict more stable configurations.

components $u^{0,1}$ and $k^{0,1}$ as $X=X_{0}+\delta X e^{-i \omega t}$, where $X_{0}$ collectively denotes the background quantities and $\delta X$ is the corresponding radial perturbation. The orthogonality conditions on $u^{\mu}$ and $k^{\mu}$ can be used to relate $\delta u^{0}, \delta k^{0}$, and $\delta k^{1}$ to the remaining functions. Radial fluid perturbations propagate at the speed $c_{s}=\sqrt{\partial P_{r} / \partial \rho}$, which is always real and subluminal for these configurations. On the other hand, the tangential speed of sound cannot be computed in our framework since it requires nonspherical perturbations.

The linear system can be reduced to a second-order differential equation for the fluid displacement, $\xi(r)=$ $i \frac{u^{r}}{\omega} e^{\nu / 2}$. The eigenvalue problem is solved by requiring $\xi(0)=0$ and $\Delta P_{r}(R)=0$, where $\Delta P_{r}=\delta p+\xi \partial_{r} P_{r}$ is the Lagrangian variation of the pressure [35]. This selects a discrete set of frequencies $\omega^{2}$, with $\omega^{2}>0\left(\omega^{2}<0\right)$ defining stable (unstable) modes.

Our results are summarized in Fig. 3, where we show the fundamental modes as a function of the compactness for representative values of $\mathcal{C}$. All of the expectations based on the mass-radius diagrams are confirmed: configurations with central densities below (above) that corresponding to the maximum mass are linearly stable (unstable). Strongly anisotropic configurations are linearly stable for $M / R \lesssim$ 0.42 , while they become linearly unstable for higher values of the compactness.

\section{EVOLUTION EQUATIONS OF ANISOTROPIC FLUIDS}

We now discuss the full nonlinear theory. The conservation of stress-energy momentum and the conservation of baryonic current,

$$
\nabla_{\mu} T^{\mu \nu}=0, \quad \nabla_{\mu}\left(\rho_{0} u^{\mu}\right)=0,
$$

imply, respectively, the conservation of energy and momentum density and the conservation of mass that govern the fluid equations.

To write this covariant conservation law as an evolution system in spherical symmetry, one needs to split the spacetime tensors and equations into their space and time components by means of the $1+1$ decomposition. The line element can be decomposed as

$$
d s^{2}=-\alpha^{2}(t, r) d t^{2}+g_{r r}(t, r) d r^{2}+g_{\theta \theta}(t, r) d \Omega^{2},
$$

where $\alpha$ is the lapse function, $g_{r r}$ and $g_{\theta \theta}$ are positive metric functions, and $d \Omega^{2}=d \theta^{2}+\sin ^{2} \theta d \varphi^{2}$ is the solid angle element. These quantities are defined on each spatial foliation $\Sigma_{t}$ with normal $n_{a}=(-\alpha, 0)$ and extrinsic curvature $K_{i j} \equiv-\frac{1}{2} \mathcal{L}_{n} \gamma_{i j}$, where $\mathcal{L}_{n}$ is the Lie derivative along $n^{a}$.

Notice that, since $\Pi_{\nu}^{\mu}=\operatorname{diag}(0,0,1,1)$ in spherical symmetry, the anisotropy function $\sigma$ enters only in $T_{\theta}^{\theta}$ and $T_{\phi}^{\phi}$, while the rest of $T_{\nu}^{\mu}$ is formally the same as for an isotropic fluid. The projections of this tensor and the baryonic current, in spherical symmetry, are given by

$$
\begin{gathered}
D=\rho_{0} W, \quad U=h W^{2}-P_{r}, \quad S_{r}=h W^{2} v_{r}, \\
S_{r}^{r}=h W^{2} v^{r} v_{r}+P_{r}, \quad S_{\theta}^{\theta}=P_{r}-\mathcal{C} \sigma,
\end{gathered}
$$

where we have defined the enthalpy $h=\rho+p=\rho_{0}(1+$ $\epsilon)+p$ in terms of the rest-mass density $\rho_{0}$ and the internal energy $\epsilon$. Furthermore, we have defined

$$
\begin{gathered}
\sigma=\rho(1+\epsilon) \frac{W}{\sqrt{g_{r r}}}\left[\frac{v_{r}}{\alpha} \partial_{t} P_{r}+\partial_{r} P_{r}\right], \\
\partial_{t} P_{r}=f\left(\alpha, g_{r r}, u^{r}, \partial u^{r}, \partial_{r} P_{r}, \partial_{r} \rho, \sigma ; \overline{\mathcal{C}}\right), \\
u^{r} \equiv W v^{r}, \quad W \equiv \frac{1}{\sqrt{1-v_{r} v^{r}}} .
\end{gathered}
$$

It is straightforward to obtain generic evolution equations (in the sense that they do not depend on the specific form of the stress-energy tensor) for these projected quantities by projecting the conserved equations (6). The evolved conserved quantities $\left\{D, U, S_{r}\right\}$ are not modified by the anisotropies. Therefore, the algorithm to convert from conserved to primitive or physical fields $\left\{\rho_{0}, \epsilon, P_{r}, v_{r}\right\}$, given an equation of state $P_{r}=P_{r}\left(\rho_{0}, \epsilon\right)$, is the same as for isotropic fluids.

Einstein's equations can be written by using the $\mathrm{Z3}$ formulation in spherical symmetry [36]. This formulation introduces independent variables in order to form a firstorder evolution system. The final system must be complemented with gauge conditions for the lapse. We use the 


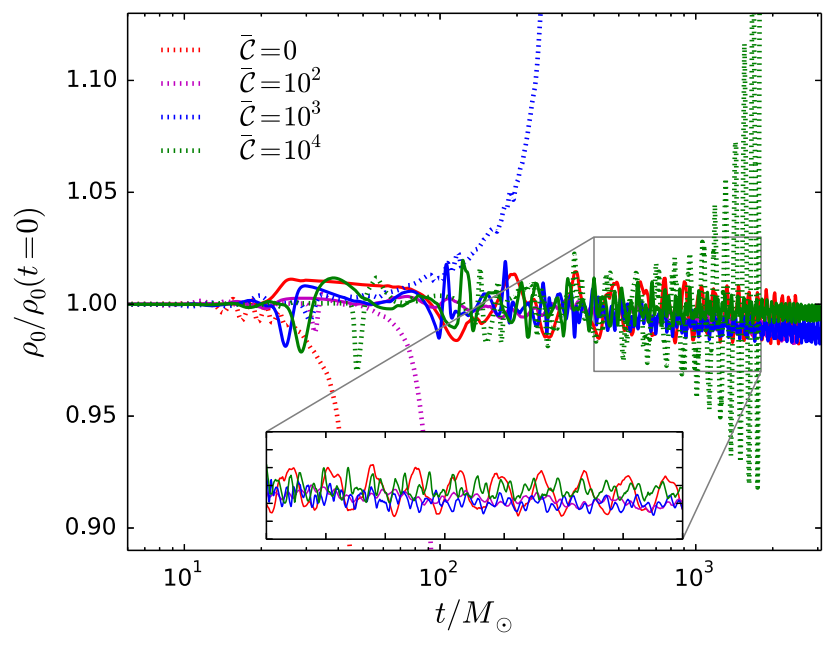

FIG. 4. Central density $\rho_{0}$ as a function of time for various configurations in the stable (continuous curves) and in the unstable (dotted curves) branch.

harmonic slicing condition $\partial_{t} \ln \alpha=-\alpha t r K$, where $\operatorname{tr} K=K_{r}^{r}+2 K_{\theta}^{\theta}$.

Finally, the evolution system is written in balance law form [37],

$$
\partial_{t} \mathbf{U}+\partial_{i} F^{i}(\mathbf{U})=G(\mathbf{U})
$$

where $\mathbf{U}=\left\{\alpha, g_{r r}, g_{\theta \theta}, K_{r}^{r}, K^{\theta}{ }_{\theta}, A_{r}, D_{r r}^{r}, D_{r \theta}^{r}, Z_{r}, D, U, S_{r}\right\}$ is a vector containing the final set of evolution fields. Further details on the numerical procedure and code validation are provided in Appendix B.

Thus, one of our main results is that the system of partial differential equations describing the anisotropic fluid and the dynamical spacetime (which are detailed above) is well behaved, and fully nonlinear simulations can be performed. Our simulations confirm the stability properties of the equilibrium configurations found in the previous section. Figure 4 displays the evolution of the central value of the rest-mass density of both stable and unstable equilibrium configurations, for different values of the parameter $\mathcal{C}$, as a function of time. Clearly, small numerical perturbations drive unstable solutions away from their original configuration, whereas they remain bound for solutions in the stable branch.

The full nonlinear results for the time scale $\tau$ are compared with the linear analysis in Fig. 3. We find good agreement for stable configurations at moderately small compactness. While our code agrees very well with the linearized analysis for $\mathcal{C}=0$, for large compactness and large values of $\overline{\mathcal{C}}$, the nonlinear evolution shows that the threshold for stability is pushed to larger compactness, as compared to linear analysis. Furthermore, although not shown in Fig. 3, we find indication that unstable configurations typically have a lifetime longer than the one predicted solely by linearized studies. We do not have a solid explanation for this discrepancy; it could be due to nonlinearities driving the energy to higher modes, or to other effects. We postpone a detailed analysis for the future, but we point out that such nonlinear results are potentially exciting: the merger of two $\mathcal{C}$-stars might form an ultracompact configuration which lies on the unstable branch, but with a long lifetime and therefore with the potential to impart unique signatures to the postmerger gravitationalwave (GW) signal, some of which are described below. In addition, $\mathcal{C}$-stars with compactness $M / R \approx 0.4$ are nonlinearly stable. To the best of our knowledge, this is the first model of stable ultracompact objects featuring a clear photon sphere (at $R=3 M$ ) and which are dynamically well behaved.

\section{PHENOMENOLOGY OF $\mathcal{C}$-STARS}

We have just shown that $\mathcal{C}$-stars can be radially stable and as compact as BHs. Thus, they can essentially mimic all of the geodesic properties of a BH [6]. Some smoking-gun signature will however appear in dynamical situations, as we now describe.

\section{A. Tidal Love numbers}

The tidal Love numbers (TLNs) define the deformability of a star immersed in an external field, such as the one produced by a companion in a binary [38]. These quantities are particularly useful for GW astronomy, since they affect the late-inspiral GW signal from a coalescence and contain information about the nature of the merging objects [39]. The prime motivation to measure TLNs is to constrain the neutron-star equation of state $[39,40]$ and to convey information on the nature of compact objects [41-44]: in GR, the TLNs of a BH are precisely zero [45-50], but they are nonvanishing for ECOs [41,51-53], being thus a smoking gun for ultracompact horizonless objects.

The TLNs can be computed with standard techniques $[38,41-43,54]$ by studying small nonspherical (quadrupolar) deformations of a compact object. As a proof of principle, we focus on the quadrupolar scalar TLNs, which are qualitatively similar to the gravitational case and provide the same phenomenology [41]. In the large-compactness limit, our results are consistent with the relation

$$
k_{2}^{\text {scalar }} \sim a \overline{\mathcal{C}}^{p}\left(\frac{\Delta}{M}\right)^{n},
$$

where $k_{2}^{\text {scalar }}$ is the scalar TLN as defined in Ref. [41], $\Delta$ is the proper distance [55] between $R$ and the Schwarzschild radius $2 M$, and $a \sim \mathcal{O}(1), p \approx 1.2$, and $n \approx(3-3.5)$ mildly depend on $\mathcal{C}$. Remarkably, this behavior is markedly different from that of other ECO models, for which the TLNs vanish logarithmically, $k_{2} \sim 1 / \log (\Delta / M)$, in the $\mathrm{BH}$ limit $[41,43]$, and shows that the TLNs of $\mathcal{C}$-stars are very small as $M / R \rightarrow 1 / 2$. As a reference, for a neutron star $k_{2}^{\text {scalar }} \approx k_{2}^{\text {gravitational }} \approx 200$ or larger [40]. 


\section{B. GW echoes}

GW echoes in the postmerger GW signal from a binary coalescence are a smoking gun for structure at the horizon scale [56-59]. Our scope here is to simply show that perturbed $\mathcal{C}$-stars produce echoes when sufficiently compact: a more detailed analysis is left for the future. We consider a test free scalar field on the background of a $\mathcal{C}$-star. Standard spherical-harmonic and Fourier decomposition lead to $\left(\partial_{x x}-\partial_{t t}-V\right) \psi(x, t)=0$, where $x$ is the tortoise coordinate defined by $d r / d x=\sqrt{-g_{00} g_{11}}$, and the effective potential reads $V(r)=-g_{00}\left(\frac{l(l+1)}{r^{2}}-\frac{g_{11}^{\prime}}{2 r g_{r r}^{2}}+\right.$ $\left.\frac{g_{00}^{\prime}}{2 r g_{11} g_{00}}\right)$, where $l=0,1,2, \ldots$ is the harmonic index and $' \equiv d / d r$. Figure 5 shows the linear response of a $\mathcal{C}$-star with initial condition $\partial_{t} \psi(x, 0)=\exp \left(-\left(x-x_{0}\right)^{2} / \sigma^{2}\right)$ and $\psi(x, 0)=0$. Echoes are associated with radiation that bounces back and forth between the object and the photon sphere [61], slowly leaking to infinity through wave tunneling [56,57]. Thus, the time delay between echoes roughly corresponds to twice the light crossing time from the center of the star to the photon sphere $[56,57,59]$,

$$
\tau_{\text {echo }}=\int_{0}^{3 M} \frac{d r}{\sqrt{e^{\nu}(1-2 m / r)}} .
$$

Interestingly, this delay time is typically dominated by the travel time within the star, not by the Shapiro delay factor $\sim \log (1-2 M / R)$ near the surface [57]. This property is akin to (isotropic) ultracompact stars near Buchdahl's limit [3,59] and to certain phenomenological models [62] considered in the past.

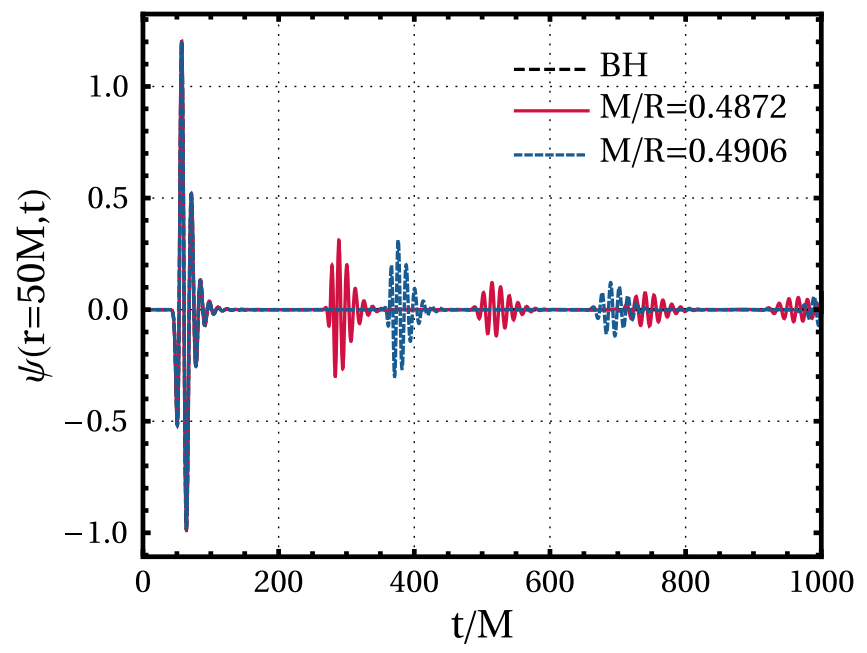

FIG. 5. GW echoes from a $\mathcal{C}$-star with $\overline{\mathcal{C}}=10^{5}$ and for two values of the compactness. We consider quadrupolar scalar perturbations with an initial Gaussian profile with parameters $x_{0}=5 M$ and $\sigma=4 M$. The corresponding response of a Schwarzschild $\mathrm{BH}$ is shown by a continuous black curve for comparison. The waveforms (normalized by their peak value) are available online [60].

\section{DISCUSSION}

To summarize, we introduced a covariant framework to study anisotropic stars in GR, whose resulting evolution system is well posed. Thus, relativistic anisotropic fluids can be explored in full-blown nonlinear evolutions, as we hope to do in the near future.

Our results are exciting: $\mathcal{C}$-stars provide a prototypical model for ultracompact objects, which is of immense utility in the quest to quantify the evidence for $\mathrm{BHs}$ and in identifying possible smoking guns for new physics $[2,3,5]$. Such configurations can be metastable and display the whole phenomenology recently predicted for ultracompact horizonless objects. In particular, they can be as massive and compact as BHs, have vanishingly small TLNs [41], and produce GW echoes [56,57] when perturbed (evading recent results [63], due to anisotropy).

It is intriguing to notice that $\mathcal{C}$-stars share many key properties with gravastars [29,31], although the dynamics of the latter lacks a solid theoretical framework (see Ref. [64] for recent progress). Thus, $\mathcal{C}$-stars might serve as an effective model for semiclassical corrections near the horizon, as predicted in other contexts $[32,65]$. Some of these models are nonperturbative in the Planck length $\ell_{P}$, and they would predict $\mathcal{C} / M^{3} \sim M / \ell_{P} \sim 10^{38}$ for $M=M_{\odot}$, which motivates the strong-anisotropy regime explored here. It is also likely that the magnitude of anisotropies grows with the compactness of the object. Anisotropic effects might become stronger during the merger and an ordinary neutron star might "anisotropize" dynamically.

We have worked with a very crude toy model. Generalizations include, for example, models with $f(\rho)=\rho^{n}$; preliminary results for $n>1$ show that stars in the stable branch are even more compact than the models presented here. We are tempted to conclude that there are very generic models of anisotropy which lead to the same phenomenology as the one we report.

Finally, we focused on the nonlinear dynamics in the spherically symmetric case; extensions of our covariant formalism to less symmetric configurations and simulations of binary $\mathcal{C}$-stars are ongoing, based on the general covariant framework presented in Appendix A. This is particularly interesting in light of our results: the massradius diagram of $\mathcal{C}$-stars suggests that, for (say) $\overline{\mathcal{C}}=10^{8}$, two merging $\mathcal{C}$-stars with equal mass $M \approx 11 M_{\odot}$ (compactness $M / R \approx 0.18$ ) might give rise to a stable $\mathcal{C}$-star near the maximum mass $M_{f} \approx 21 M_{\odot}$ and with compactness $M_{f} / R \approx 0.43$, being thus a viable candidate for an $\mathrm{ECO}+$ ECO $\rightarrow$ ECO coalescence.

\section{ACKNOWLEDGMENTS}

We thank Sante Carloni, Kento Yagi, and Stoytcho Yazadjiev for useful comments and feedback. We are grateful to Kinki University in Osaka for kind hospitality while this work began. The authors thank the Yukawa 
Institute for Theoretical Physics at Kyoto University and the Galileo Galilei Institute in Florence, where this work was completed during the YITP-T-18-05 workshop on "Dynamics in Strong Gravity Universe" and the "Fundamental Physics with LISA" workshop, respectively. P. P. acknowledges financial support provided under the European Union's H2020 ERC, Starting Grant Agreement No. DarkGRA-757480 and the kind hospitality of the Universitat de les Illes Balears, where part of this work was performed. C. P. and M. B. acknowledge support from the Spanish Ministry of Economy and Competitiveness Grants No. AYA2016-80289-P (AEI/FEDER, UE) and No. AYA2017-82089-ERC. C. P. also acknowledges support from the Spanish Ministry of Education and Science through a Ramon y Cajal grant. M. B. would like to thank CONICYT Becas Chile (Concurso Becas de Doctorado en el Extranjero) for financial support. M. B. also acknowledges the support of the PHAROS COST Action (CA16214). V. C. acknowledges financial support provided under the European Union's H2020 ERC Consolidator Grant "Matter and strong-field gravity: New frontiers in Einstein's theory," Grant Agreement no. MaGRaTh646597. This project has received funding from the European Union's Horizon 2020 research and innovation programme under the Marie Sklodowska-Curie Grant Agreement No. 690904. We acknowledge financial support provided by FCT/Portugal through Grant No. PTDC/MATAPL/30043/2017. We acknowledge the SDSC Comet and TACC Stampede2 clusters through NSF-XSEDE Award No. PHY-090003, as well as MareNostrum and the technical support provided by the Barcelona Supercomputing Center (AECT-2018-1-0003). The authors would like to acknowledge networking support by the GWverse COST Action CA16104, "Black holes, gravitational waves and fundamental physics." We acknowledge support from the Amaldi Research Center funded by the MIUR program "Dipartimento di Eccellenza" (CUP: B81I18001170001).

\section{APPENDIX A: GENERAL COVARIANT FRAMEWORK BEYOND SPHERICAL SYMMETRY}

In this appendix we generalize the covariant framework for anisotropic fluids in GR without any special symmetry. In the most general case, the pressure can be different along three generic spatial directions, identified by a triad of unit space-like vectors: $k_{\mu}, \xi_{\mu}$, and $\eta_{\mu}$. The anisotropies can be defined in terms of deviations $\sigma_{1}, \sigma_{2}$, and $\sigma_{3}$ from a reference isotropic pressure function, $P_{\text {ISO }}$. The stressenergy tensor describing the resulting anisotropic fluid can be written as

$$
T_{\mu \nu}=T_{\mu \nu}^{\mathrm{ISO}}+\sigma_{1} k_{\mu} k_{\nu}+\sigma_{2} \xi_{\mu} \xi_{\nu}+\sigma_{3} \eta_{\mu} \eta_{\nu},
$$

where we have defined the stress-energy tensor of an ordinary isotropic fluid as

$$
T_{\mu \nu}^{\mathrm{ISO}}=\left(\rho+P_{\mathrm{ISO}}\right) u_{\mu} u_{\nu}+g_{\mu \nu} P_{\mathrm{ISO}},
$$

with $u_{\mu}$ being the usual fluid four-velocity.

The unit vectors $k_{\mu}, \xi_{\mu}$, and $\eta_{\mu}$ are orthogonal to each other and to $u_{\mu}$, i.e., they are constrained by the nine conditions $k^{\mu} k_{\mu}=\xi^{\mu} \xi_{\mu}=\eta^{\mu} \eta_{\mu}=1$ and $u^{\mu} k_{\mu}=u^{\mu} \xi_{\mu}=$ $k^{\mu} \xi_{\mu}=k^{\mu} \eta_{\mu}=\xi^{\mu} \eta_{\mu}=u^{\mu} \eta_{\mu}=0$. These conditions can be used to fix nine out of the $3 \times 4$ components of $k_{\mu}$, $\xi_{\mu}$, and $\eta_{\mu}$. The remaining three components can be arbitrarily fixed without loss of generality, since they are associated with the translation of the origin of the frame identified by the triad of pressure vectors.

Using the above conditions, we can define the projections

$$
\begin{gathered}
u^{\mu} T_{\mu \nu}=\rho u_{\nu}, \\
k^{\mu} T_{\mu \nu}=\left(P_{\mathrm{ISO}}+\sigma_{1}\right) k_{\nu}=P_{1} k_{\nu}, \\
\xi^{\mu} T_{\mu \nu}=\left(P_{\mathrm{ISO}}+\sigma_{2}\right) \xi_{\nu}=P_{2} \xi_{\nu}, \\
\eta^{\mu} T_{\mu \nu}=\left(P_{\mathrm{ISO}}+\sigma_{3}\right) \eta_{\nu}=P_{3} \eta_{\nu},
\end{gathered}
$$

where we defined $P_{i}:=P_{\text {ISO }}+\sigma_{i}$. Thus, in this notation each vector is related with a specific direction of anisotropy. When $\sigma_{1}=\sigma_{2}=\sigma_{3}=0$ the stress-energy tensor above reduces to the usual stress-energy tensor for an isotropic perfect fluid.

In analogy to the spherically symmetric case, we provide three equations of state for $P_{1}, P_{2}$, and $P_{3}$ of the form

$$
\begin{aligned}
& \sigma_{1}=P_{1}-P_{\mathrm{ISO}}=\mathcal{C}_{1} f_{1}(\rho) k^{\mu} \nabla_{\mu} \rho, \\
& \sigma_{2}=P_{2}-P_{\mathrm{ISO}}=\mathcal{C}_{2} f_{2}(\rho) \xi^{\mu} \nabla_{\mu} \rho, \\
& \sigma_{3}=P_{3}-P_{\mathrm{ISO}}=\mathcal{C}_{3} f_{3}(\rho) \eta^{\mu} \nabla_{\mu} \rho,
\end{aligned}
$$

where the free constants $\mathcal{C}_{1}, \mathcal{C}_{2}$, and $\mathcal{C}_{3}$ are generically dimensionful. At variance with the main text, we have defined the equations of state in terms of covariant derivatives of the density, since the latter is unique.

Let us now show that the general framework reduces to the spherically symmetric case considered in the main text. In spherical symmetry, the angular components of the vectors must vanish. In this case, $k_{\mu}=\left(k_{0}, k_{1}, 0,0\right)$ and the other two vectors are identically zero, $\xi_{\mu}=0$ and $\eta_{\mu}=0$. Equations (A7)-(A9) then take the form

$$
\begin{gathered}
P_{1}=P_{\mathrm{ISO}}+\mathcal{C}_{1} f_{1}(\rho) k^{\mu} \nabla_{\mu} \rho, \\
P_{2}=P_{\mathrm{ISO}}, \\
P_{3}=P_{\mathrm{ISO}},
\end{gathered}
$$


and therefore $\sigma_{2}=\sigma_{3}=0$. The spherically symmetric case is recovered by defining $P_{t}=P_{\mathrm{ISO}}, P_{t}=P_{r}-$ $\mathcal{C}_{1} f_{1}(\rho) k^{\mu} \nabla_{\mu} \rho$, and $f_{1}(\rho)=f(\rho) \partial P_{r} / \partial \rho$ to account for the different definition in the equation of state.

\section{APPENDIX B: CODE VALIDATION}

The numerical code used in this work is a simple extension of the one presented in Ref. [37] to study fermionboson stars. The spatial discretization of spacetime fields is performed using a third-order accurate finite volume method [66], which can be viewed as a fourth-order finitedifference scheme plus third-order adaptive dissipation. The dissipation coefficient is given by the maximum propagation speed in each grid point. For the fluid matter fields, we use a high-resolution shock capturing method with monotonic-centered limiter. The time evolution is performed through the method of lines using a third-order accurate strong stability-preserving Runge-Kutta integration scheme, with a Courant factor of $\Delta t / \Delta r=0.2$ so that the Courant-Friedrichs-Levy condition dictated by the principal part of the equations is satisfied. Most of the simulations presented in this work have been done with a spatial resolution of $\Delta r=0.00625 M_{\odot}$, in a domain with outer boundary situated at $r=100 M_{\odot}$. We have verified, by changing the position of the outer boundary, that the results do not vary significantly with different choices of the boundary. We use maximally dissipative boundary conditions for the spacetime variables, and outflow boundaries for the fluid matter fields.

This nonlinear code has passed a large set of stringent tests. First, as it was shown in Fig. 9 of Ref. [37], it already recovered the well-known frequencies of a neutron star with mass $M=1.4 M_{\odot}$ (as calculated, for instance, in Ref. [67]). Furthermore, we have extensively compared our nonlinear and linear codes for $\overline{\mathcal{C}}=0$, finding an excellent agreement on the quasinormal frequencies, as shown in Table II.

Finally, we always performed convergence tests, especially for the extreme cases with $\overline{\mathcal{C}} \gg 1$. Indeed, in the latter cases passing convergence tests requires unusually high spatial/time resolutions to resolve steep structures near the radius of the star (see below). For all stable cases we found

TABLE II. Characteristic oscillation times for $\overline{\mathcal{C}}=0$ and different compactness. We compare the result of the nonlinear simulations (second column) with those of the linear analysis (third column).

\begin{tabular}{lcc}
\hline \hline$M / R$ & $\tau_{\text {nonlinear }}$ & $\tau_{\text {linear }}$ \\
\hline 0.12 & 22.3 & 22.5 \\
0.14 & 22.4 & 22.3 \\
0.16 & 23.06 & 22.9 \\
0.18 & 24.94 & 25.2 \\
0.20 & 34.75 & 34.6 \\
\hline \hline
\end{tabular}

that the results converge as expected. The frequencies reported in the main text are obtained with the highest resolution and we checked that they are almost insensitive to the time/spatial resolution.

We display the central density of the star for one of these convergence tests in Fig. 6, corresponding to a configuration with $M / R=0.306$ with $\overline{\mathcal{C}}=1000$. We use $\Delta r=$ $\{0.00625,0.003125,0.0015625\} M_{\odot}$ for this test. The star is initially in equilibrium, only perturbed by numerical discretization errors, and oscillates with its associated normal frequencies. In addition, there is a deviation from the constant stationary value due to numerical errors, which decreases as the resolution is increased. The solution shows almost a second convergence. Notice that, although this value is below the third-order convergence expected for smooth solutions, it is higher than the linear convergence expected in the presence of strong shocks.
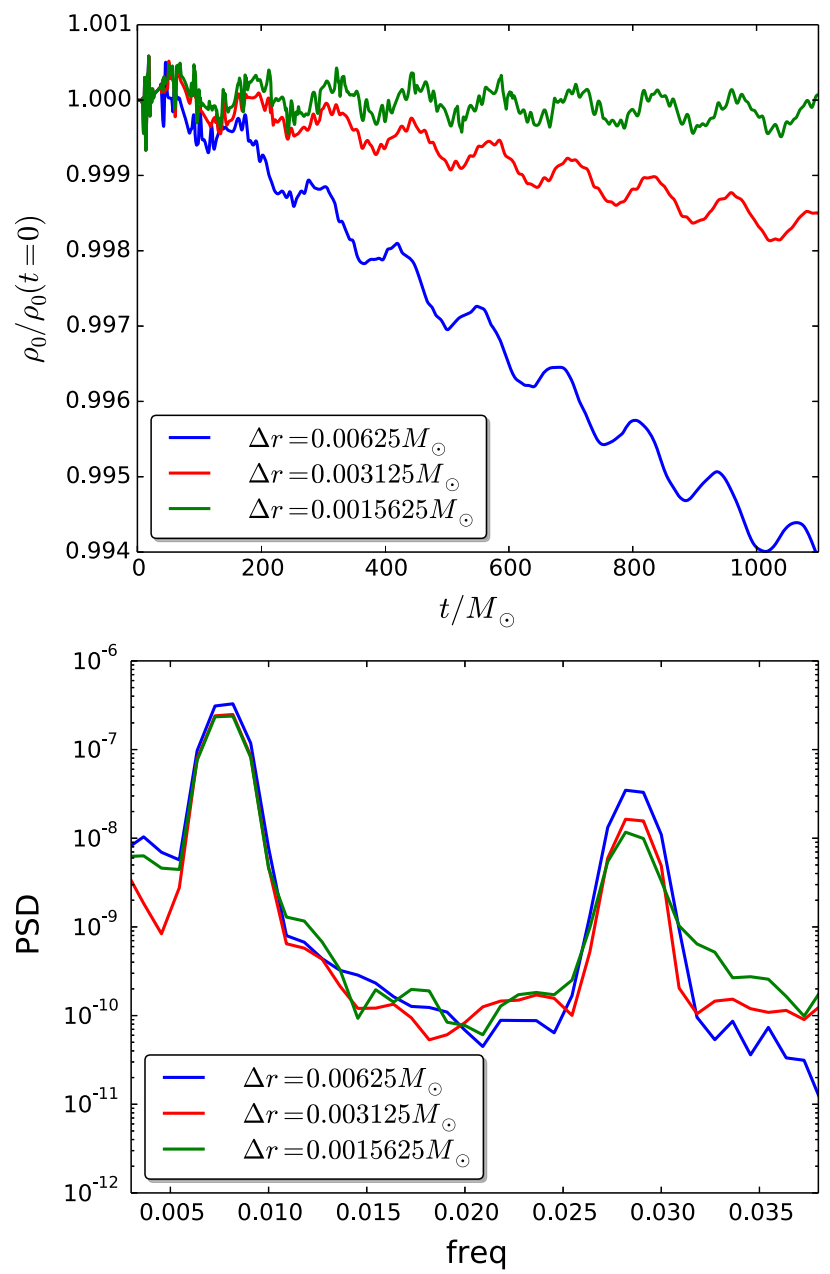

FIG. 6. Convergence test for $\overline{\mathcal{C}}=1000$ and $M / R \approx 0.306$. (Top) Central density $\rho_{0}$ as a function of time for three resolutions $\Delta r=\{0.00625,0.003125,0.0015625\} M_{\odot}$. (Bottom) Fourier transform of the central density. The first peak is centered at the same frequency (i.e., corresponding to the fundamental mode of the characteristic oscillation) for the three resolutions. 
[1] H. A. Buchdahl, Phys. Rev. 116, 1027 (1959).

[2] V. Cardoso and P. Pani, Nat. Astron. 1, 586 (2017).

[3] V. Cardoso and P. Pani, arXiv:1904.05363.

[4] L. Barack et al., arXiv:1806.05195.

[5] R. Carballo-Rubio, F. Di Filippo, S. Liberati, and M. Visser, Phys. Rev. D 98, 124009 (2018).

[6] V. Cardoso and P. Pani, arXiv:1904.05363.

[7] R. Kippenhahn, A. Weigert, and A. Weiss, Stellar Structure and Evolution (Springer, New York, 2012).

[8] M. Ruderman, Annu. Rev. Astron. Astrophys. 10, 427 (1972).

[9] V. Canuto and S. M. Chitre, Phys. Rev. D 9, 1587 (1974).

[10] S. L. Liebling and C. Palenzuela, Living Rev. Relativity 15, 6 (2012).

[11] R. F. Sawyer and D. J. Scalapino, Phys. Rev. D 8, 1260 (1973).

[12] B. Carter and D. Langlois, Nucl. Phys. B531, 478 (1998).

[13] R. L. Bowers and E. P. T. Liang, Astrophys. J. 188, 657 (1974).

[14] P. S. Letelier, Phys. Rev. D 22, 807 (1980).

[15] S. S. Bayin, Phys. Rev. D 26, 1262 (1982).

[16] K. Dev and M. Gleiser, Gen. Relativ. Gravit. 34, 1793 (2002).

[17] M. K. Mak and T. Harko, Proc. R. Soc. A 459, 393 (2003).

[18] K. Dev and M. Gleiser, Gen. Relativ. Gravit. 35, 1435 (2003).

[19] L. Herrera, A. Di Prisco, J. Martin, J. Ospino, N. O. Santos, and O. Troconis, Phys. Rev. D 69, 084026 (2004).

[20] H. Andreasson, J. Differ. Equations 245, 2243 (2008).

[21] W. Hillebrandt and K. O. Steinmetz, Astron. Astrophys. 53, 283 (1976).

[22] D. D. Doneva and S. S. Yazadjiev, Phys. Rev. D 85, 124023 (2012).

[23] H. O. Silva, C. F. B. Macedo, E. Berti, and L. C. B. Crispino, Classical Quantum Gravity 32, 145008 (2015).

[24] K. Yagi and N. Yunes, Phys. Rev. D 91, 123008 (2015).

[25] K. Yagi and N. Yunes, Phys. Rev. D 91, 103003 (2015).

[26] K. Yagi and N. Yunes, Classical Quantum Gravity 33, 095005 (2016).

[27] B. Biswas and S. Bose, Phys. Rev. D 99, 104002 (2019).

[28] S. Carloni and D. Vernieri, Phys. Rev. D 97, 124057 (2018).

[29] P. O. Mazur and E. Mottola, Proc. Natl. Acad. Sci. U.S.A. 101, 9545 (2004).

[30] M. Visser and D. L. Wiltshire, Classical Quantum Gravity 21, 1135 (2004).

[31] C. Cattoen, T. Faber, and M. Visser, Classical Quantum Gravity 22, 4189 (2005).

[32] P. O. Mazur and E. Mottola, Classical Quantum Gravity 32, 215024 (2015).

[33] C. A. Kolassis, N. O. Santos, and D. Tsoubelis, Classical Quantum Gravity 5, 1329 (1988).

[34] S. L. Shapiro and S. A. Teukolsky, Black holes, white dwarfs, and neutron stars: The physics of compact objects (Wiley, New York, 1983).

[35] K. Kokkotas and J. Ruoff, Astron. Astrophys. 366, 565 (2001).

[36] A. Bernal, J. Barranco, D. Alic, and C. Palenzuela, Phys. Rev. D 81, 044031 (2010).
[37] S. Valdez-Alvarado, C. Palenzuela, D. Alic, and L. A. Ureña-López, Phys. Rev. D 87, 084040 (2013).

[38] E. Poisson and C. Will, Gravity: Newtonian, PostNewtonian, Relativistic (Cambridge University Press, Cambridge, England, 2014).

[39] E. E. Flanagan and T. Hinderer, Phys. Rev. D 77, 021502 (2008).

[40] B. P. Abbott et al. (Virgo and LIGO Scientific Collaborations), Phys. Rev. Lett. 121, 161101 (2018).

[41] V. Cardoso, E. Franzin, A. Maselli, P. Pani, and G. Raposo, Phys. Rev. D 95, 084014 (2017).

[42] N. Sennett, T. Hinderer, J. Steinhoff, A. Buonanno, and S. Ossokine, Phys. Rev. D 96, 024002 (2017).

[43] A. Maselli, P. Pani, V. Cardoso, T. Abdelsalhin, L. Gualtieri, and V. Ferrari, Phys. Rev. Lett. 120, 081101 (2018).

[44] N. K. Johnson-Mcdaniel, A. Mukherjee, R. Kashyap, P. Ajith, W. Del Pozzo, and S. Vitale, arXiv:1804.08026.

[45] T. Binnington and E. Poisson, Phys. Rev. D 80, 084018 (2009).

[46] T. Damour and A. Nagar, Phys. Rev. D 80, 084035 (2009).

[47] H. Fang and G. Lovelace, Phys. Rev. D 72, 124016 (2005).

[48] N. Gürlebeck, Phys. Rev. Lett. 114, 151102 (2015).

[49] E. Poisson, Phys. Rev. D 91, 044004 (2015).

[50] P. Pani, L. Gualtieri, A. Maselli, and V. Ferrari, Phys. Rev. D 92, 024010 (2015).

[51] P. Pani, Phys. Rev. D 92, 124030 (2015); 95, 049902(E) (2017).

[52] N. Uchikata, S. Yoshida, and P. Pani, Phys. Rev. D 94, 064015 (2016).

[53] R. A. Porto, Fortschr. Phys. 64, 723 (2016).

[54] T. Hinderer, Astrophys. J. 677, 1216 (2008); 697, 964(E) (2009).

[55] A. Maselli, P. Pani, V. Cardoso, T. Abdelsalhin, L. Gualtieri, and V. Ferrari, arXiv:1811.03689.

[56] V. Cardoso, E. Franzin, and P. Pani, Phys. Rev. Lett. 116, 171101 (2016).

[57] V. Cardoso, S. Hopper, C. F. B. Macedo, C. Palenzuela, and P. Pani, Phys. Rev. D 94, 084031 (2016).

[58] V. Ferrari and K. D. Kokkotas, Phys. Rev. D 62, 107504 (2000).

[59] P. Pani and V. Ferrari, Classical Quantum Gravity 35, 15LT01 (2018).

[60] http://www.darkgra.org.

[61] V. Cardoso, L. C. B. Crispino, C. F. B. Macedo, H. Okawa, and P. Pani, Phys. Rev. D 90, 044069 (2014).

[62] E. Barausse, R. Brito, V. Cardoso, I. Dvorkin, and P. Pani, Classical Quantum Gravity 35, 20LT01 (2018).

[63] A. Urbano and H. Veermäe, J. Cosmol. Astropart. Phys. 04 (2019) 011.

[64] P. Beltracchi and P. Gondolo, Phys. Rev. D 99, 044037 (2019).

[65] R. Carballo-Rubio, Phys. Rev. Lett. 120, 061102 (2018).

[66] D. Alic, C. Bona, C. Bona-Casas, and J. Massó, Phys. Rev. D 76, 104007 (2007).

[67] J. A. Font, T. Goodale, S. Iyer, M. Miller, L. Rezzolla, E. Seidel, N. Stergioulas, W. M. Suen, and M. Tobias, Phys. Rev. D 65, 084024 (2002). 